\title{
Timpanoplastías en pacientes mayores de 50 años. Experiencia en el Hospital San Juan de Dios
}

\author{
Tympanoplasty in patients older than $\mathbf{5 0}$ years old. \\ San Juan de Dios Hospital experience
}

Loreto Nicklas $D^{1}$, Saúl Salgado $C^{1}$, Margarita Arancibia $S^{1}$, Juan José Orellana $C^{2}$.

\section{RESUMEN}

Introducción: Se ha descrito que el resultado de las cirugías de la membrana timpánica está condicionado por la edad del paciente, siendo peor en los casos de edades extremas. Según las últimas publicaciones esta tendencia se ha demostrado en disminución.

Objetivo: Evaluar los resultados anatómicos y auditivos de las timpanoplastías de nuestro servicio en pacientes mayores de 50 años, en un periodo de 4 años, analizando además otras variables relativas a la cirugía

Material y método: Revisión de 23 oídos.

Resultados: Éxito anatómico en el 82,6\% de los casos con una primera cirugía En el $52 \%$ se obtuvo mejoría auditiva en al menos $10 \mathrm{~dB}$ del promedio tonal puro aéreo.

Conclusión: No hay diferencias significativas anatómicas y auditivas en estegrupo de edad con respecto al resto de la población general ni tampoco en subgrupos de edades más extremas. Tampoco se encontraron diferencias significativas en relación al tipo de injerto, posición de éste y vía de abordaje utilizada, ni en relación a variables quirúrgicas como son: uso de antibiótico posoperatorio, presencia de miringoesclerosis, uso de gelfoam en la caja y estado del oído contralateral.

Palabras Clave: Timpanoplastías, resultados, adultos mayores.

\section{ABSTRACT}

Background: It has been published that the result of tympanic membrane surgery is conditioned by the patient's age, being of worse prognosis in elder patients. According to recent studies, this tendency is decreasing.

Aim: To assess the anatomical and auditory results of tympanoplasty performed in our department on patients older than 50 years old, in a 4-year period, analyzing other variables as well.

Methods: Study of 23 patients.

Results: The surgery was anatomically succesful in $82,6 \%$ of cases after a first surgery. In $52 \%$ of patients there was an auditory improvement of at least $10 \mathrm{~dB}$ of the aerial pure tone average.

Conclusions: There were no significant anatomical or auditory differences in this age

${ }^{1}$ Médicos del Servicio de Otorrinolaringología, Hospital San Juan de Dios.

Bioestadístico, Departamento de Epidemiología, Facultad de Medicina de la Universidad de la Frontera, Temuco. 
group compared with the general population, nor in the subgroup of older age. Moreover, there were no significant differences related to the type of graft, its position or surgical technique used, nor related to surgical variables such as use of postoperative antibiotic, presence of myringosclerosis, use of gelfoam or status of the contralateral ear.

Key words: Tympanoplasty, results, elder patients.

\section{INTRODUCCIÓN}

日 resultado de las cirugías de la membrana timpánica está condicionado por la edad del paciente según algunos autores, siendo éste más adverso en los extremos de la vida. Sin embargo, existe poca literatura que respal de esta aseveración y los malos resultados serían más bien desde el punto de vista auditivo que anatómico ${ }^{1,2}$. Al contrario existen trabajos que demuestran cambios posquirúrgicos satisfactorios en sus series ${ }^{3-7}$.

En el caso de los adultos mayores de 50 años, el resultado estaría condicionado por el deterioro en la cicatrización que ocurre con el envejecimiento de los tejidos además de la concomitancia de ciertas patologías con alteración de la microcirculación o microangiopatías que son más prevalentes a mayor edad.

Las ventajas que trae cerrar la cavidad timpánica para este grupo de pacientes, han supe rado estos factores de mal pronóstico y por otra parte los avances médicos permiten mejor control perioperatorio de estos pacientes. Debemos tomar en cuenta el envejecimiento progresivo de la población al mejorar las expectativas de vida, por lo que en un futuro próximo estaremos controlando un gran volumen de pacientes mayores de 50 años.

En publicaciones anteriores en Revista de Corrinolaringología y Grugía de Cabeza y arello, en relación a resultados de timpanoplastías en pacientes mayores de 50 años, podemos mencionar a Plaza y Ote $^{8}$ quienes en 1980 en un total de 15 pacientes mayores de 50 años encontraron éxito en $58 \%$ que disminuía a 33\% en los mayores de 60 años. En el mismo trabajo se encontró en los menores de 50 años (total de 339 casos en estudio) $49,5 \%$ de éxito anatómico.

En el 2001 Vergara y col$^{9}$ comunican resultados de timpanoplastías en todas las edades ( $90 \%$ de éxito anatómico), con 6 casos mayores de 50 años y un éxito de $50 \%$ en ese grupo, sin embargo se debe notar que son pocos casos. Fnalmente en el 2003, Vallejos y collo en un total de 118 cirugías en mayores de 50 años encontraron un éxito anatómico del $79,9 \%$, con peores resultados en los mayores de 60 años, lo que fue estadísticamente significativo, pero sin embargo los resultados eran suficientemente buenos como para que, a pesar de lo encontrado, se recomendara la cirugía en este grupo etáreo.

日 objetivo principal de éste trabajo es evaluar los resultados anatómicos y auditivos en pacientes mayores de 50 años después de una cirugía de reparación de la membrana timpánica y analizar el impacto de la edad en este grupo de pacientes. También analizaremos la relación del resultado con variables relativas a la técnica quirúrgica empleada y a condiciones preoperatorias del paciente que pudieran alterar los resultados.

\section{MATERIAL Y MÉTODO}

Se realizó un estudio retrospectivo analizando las fichas clínicas de pacientes sometidos por primera vez a cirugías de cierre de perforaciones de membrana timpánica, realizadas en el Servicio de Otorrinolaringología del Hospital San Juan de Dios en pacientes de 50 años o más, entre los años 1998 y 2002. Se excluyeron aquellos con indicación quirúrgica por sospecha de colesteatoma.

Se exigió un seguimiento mínimo posoperatorio de al menos 10 meses y los pacientes que no lo cumplían fueron citados para reevaluación. En total se revisaron 23 oídos, todos con seguimiento completo en donde se efectuó cierre de membrana timpánica

Se analizaron los resultados anatómicos y funcionales y se efectuó un análisis estadístico con Stata 8.0.

Se definió como éxito anatómico al injerto íntegro hasta el final del seguimiento, y como resultado satisfactorio auditivo aquellos casos que mantienen la audición preoperatoria o la mejoran, 
en otras palabras que el Promedio Tonal Puro (PTP) aéreo varíe en $+10 \mathrm{~dB}$ o -10 dB, o lo mejore en más de $10 \mathrm{~dB}$, respectivamente. Sin compromiso del umbral óseo evidenciable en audiometría.

\section{RESULTADOS}

En los pacientes mayores de 50 años hubo un total de 23 oídos en 21 pacientes. Dos casos fueron bilatera les. La edad mediana fue de 57 años con un rango entre 50 a 73 años. La mayor parte de nuestros pacientes eran menores de 59 años (Figura 2).

日 promedio de seguimiento de los casos fue de 32,3 meses con un rango de 15 a 60 meses. Trece por ciento ( 3 casos) de los pacientes tenían antecedentes mórbidos concomitantes como Diabetes Mellitus tipo 2, Hipertensión Arterial y Limitación Orónica del Hujo Aéreo, ninguno de los cuales fue necesario reoperar. En esta casuística no hay pacientes con enfermedades importantes que contraindiquen la anestesia ge neral y tampoco se detectaron otras enfermedades con repercusión en la microcirculación.

De los tipos de cirugías realizadas la mayoría fueron timpanoplastías destacando en ellas $5 \mathrm{ca}$ sos tipo III, el resto miringoplastías y cierres de caja (Figura 1), estos últimos definidos como el cierre de perforaciones timpánicas en las que no se reparó la cadena dada que ésta se encontró fija por timpanoesclerosis masiva.

La vía de abordaje más frecuente fue retroauricular con 20 casos.

En relación a los resultados anatómicos (Tabla 1) se observó un éxito general de 82,6\%, con sólo 4 casos perforados después de la cirugía, de éstos, 2 se reoperaron con éxito, alcanzando un éxito con la segunda cirugía de 91,3\%. Dos casos están pendientes de reoperar.
A analizar los resultados separando en subgrupos de edad vemos que $100 \%$ de los injertos en pacientes mayores de 60 años (un total de 8) están íntegros después de la primera cirugía, en cambio en los menores de 60 años se logró $73 \%$ de éxito. Sin embargo al analizar estos resultados con el test exacto de Fisher encontramos un $p=0,154$ que no es significativo.

En relación a los resultados auditivos (Tabla 2) podemos apreciar $95,6 \%$ de resultado satisfacto-

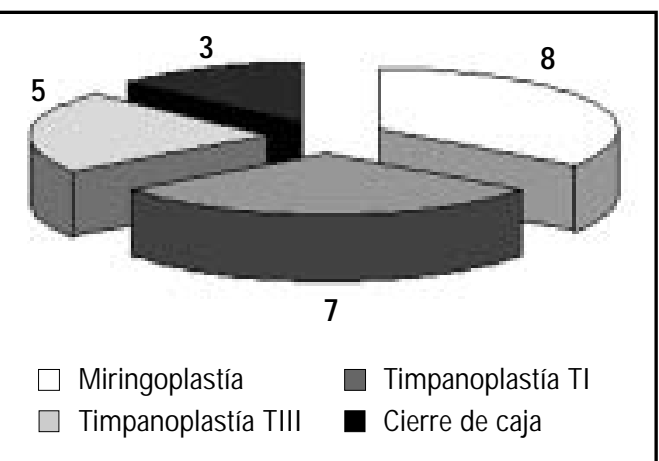

Figura 1. Tipos de cirugías en adultos mayores de 50 años.

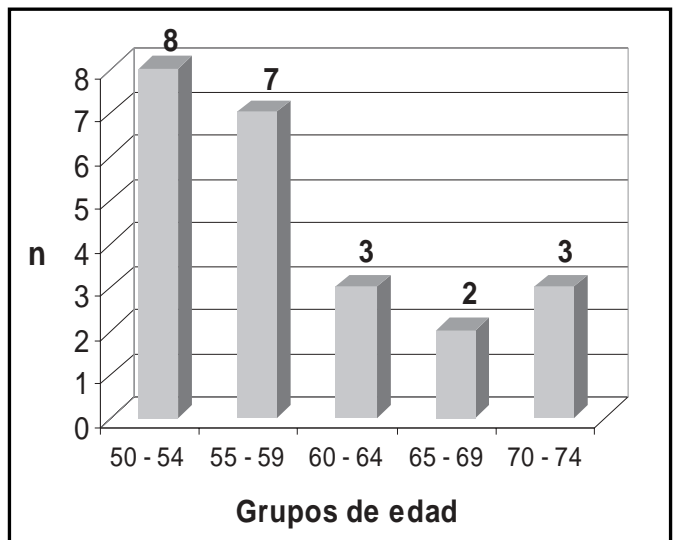

Figura 2. Distribución de los sujetos mayores de 50 años según la edad.

Tabla 1. Resultados anatómicos según la edad en mayores de 50 años

\begin{tabular}{|lcccc|}
\hline & № & Perforados & Integros & \% Integros \\
\hline 50 a 59 años & 15 & 4 & 11 & $73 \%$ \\
Más de 60 años & 8 & 0 & 8 & $100 \%$ \\
Total & 23 & 4 & 19 & $82,6 \%$ \\
\hline
\end{tabular}


Tabla 2. Resultados auditivos según la edad en mayores de 50 años

\begin{tabular}{|lcccc|}
\hline & Mejor & Igual & Peor & $\%$ Buen Resultado \\
\hline 50 a 59 años & 9 & 6 & 0 & $100 \%$ \\
Más de 60 años & 3 & 4 & 1 & $87,5 \%$ \\
Total & $12(52 \%)$ & 10 & 1 & $95,6 \%$ \\
\hline
\end{tabular}

rio, y de ellos $52 \%$ con franca mejoría de su PTP aéreo. No hubo diferencias significativas entre los subgrupos menores y mayores de 60 años. Sólo un oído está peor auditivamente (en el grupo mayor de 60 años).

En todos los casos los PIP óseos posoperatorios no disminuyeron, al contrario tendieron a mejorar en aproximadamente $4 \mathrm{~dB}$ sobre el preoperatorio.

A analizar los resultados auditivos según el tipo de cirugía tenemos que 4 de 5 casos (80\%) sometidos a timpanoplastía tipo III mejoró su audición en más de $10 \mathrm{~dB}$. y 4 de 9 casos de miringoplastías (44.4\%) mejoraron su audición. Solo un caso empeoró su audición, y correspondió a un cierre de caja

En cuanto a la técnica de colocación del injerto sobre el remanente timpánico medial o lateral a éste, el tipo de injerto: fascia temporal o pericondrio tragal y la vía de abordaje endoaural o retroauricular no hubo diferencias significativas en cuanto al éxito anatómico y auditivo, cabe destacar que mayoritaria mente se utilizaron injertos de fascia temporal, con técnica lateral a la membrana y por vía retroauricular (Figura3).

Gras variables que pudieran alterar los resultados de la cirugía fueron analizados, dentro de estos el uso de antibiótico tópico posoperatorio sobre el injerto que en teoría evitaría la infección y por consecuencia la perforación de éste, no encontrándose diferencias en relación a su uso o no en relación al resultado anatómico $(p=0,46)$. Tampoco hubo diferencias en los casos en que el cirujano estaba en formación ( $p$ $=0,667$ ). Sobre la mejoría auditiva, el uso de gelfoam en caja, que pudieraser un factor productor de bridas y por lo tanto alterar los resultados posoperatorios, tampoco tuvo diferencias significativas $(p=0,31)$, lo mismo se evidenció en relación a la presencia de miringoesclerosis ( $p=0,7$ ) y tampoco hubo relación con el estado del oído contralateral $(p=0,79)$. En este punto es importante mencionar que 10 de 23 casos tenían el oído contralateral sano (43\%), 10 casos tenían su oído contralateral perforado y 2 casos pre sentaban colesteatoma previamente operado en el otro oído (8,7\%).

\section{DISCUSIÓN}

Así como hasido descrito en otros trabajos realizados en diversos centros y publicados en nuestra revista, el buen resultado anatómico obtenido en nuestra serie

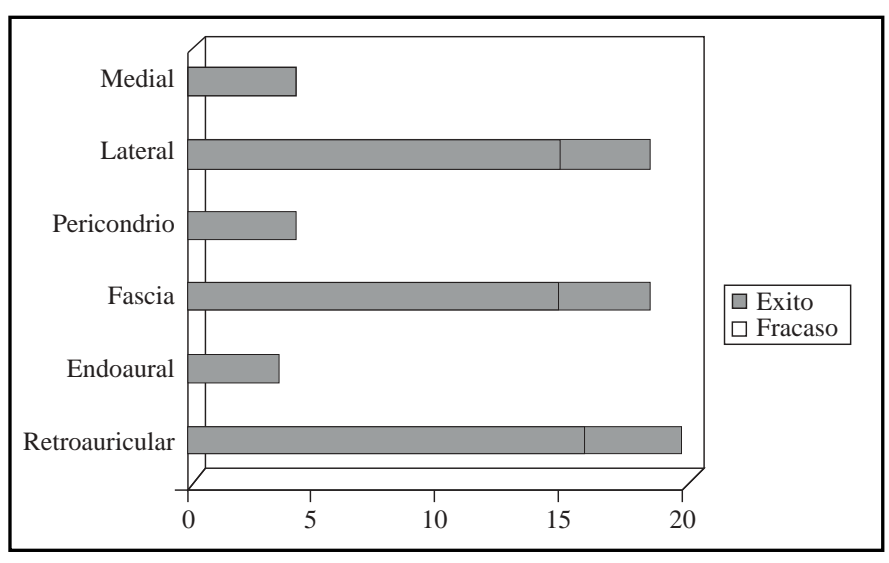

Figura 3. Resultados anatómicos y auditivos según variables quirúrgicas en mayores de 50 años. 
de pacientes es satisfactorio. Los casos estudiados son pacientes en los que se pensaba que la edad y los factores relacionados a ésta podían influir en el resultado, y por lo tanto existía cierta tendencia a no operarlos. Mediante estos resultados es posible desmitificar este aspecto y por lo tanto indicar con mayor libertad cirugías que sólo pudiesen estar contraindicada por factores relativos a la anestesia.

En nuestros pacientes se logró éxito anatómico en el $82 \%$ de los adultos, porcentaje que aumenta al indicar una reoperación encontrando que los 2 casos reperforados se han reoperado satisfactoriamente. $\mathrm{\theta}$ éxito reportado en nuestro mismo centro hospitalario para pacientes de todas las edades es de $90 \%$, cifra similar a la reportada en este estudio ${ }^{9}$.

En el $52 \%$ de los oídos se obtuvo mejoría auditiva en 10dB del PIP aéreo preoperatorio, lo quees bastante bueno en relación a lo reportado previamente ${ }^{10}$.

Las timpanoplastías III tienen buen resultado auditivo en adultos en un $80 \%$ (5 casos), lo que es comparable con los resultados publicados obtenidos en cirugías por patología peligrosa con reparación de la cadena ${ }^{11}$.

No encontramos diferencias anatómicas ni auditivas entre los menores de 59 años y los mayores de 60 años, pero se debe considerar en este trabajo $\mathrm{el}$ bajo tamaño muestral en el extremo de edad.

No hubo diferencias estadísticas en el éxito anatómico y auditivo en relación al tipo de injerto, posición de éste con respecto al remanente timpánico y vía de abordaje en los dos grupos.

Tampoco se evidenció relación entre el resultado posoperatorio y el uso de antibiótico, presencia de miringoesclerosis, uso de gelfoam en caja y el estado del oído contralateral.

Nuestro desafío es aumentar este tipo de cirugías en este grupo de pacientes y continuar analizando variables que permitan mejores resultados, sin embargo en nuestro centro la patología ótica peligrosa continúa ocupando el mayor quehacer como otólogos.

Como recomendación es posible esperar bue nos resultados anatómicos y funcionales en adul- tos mayores de 50 años y por esto se deberían realizar timpanoplastías en este grupo de pacientes, si las condiciones generales no lo impiden.

\section{BIBLIOGRAFÍA}

1. RAPDOUR S. Tympanoplasty in geriatric patients: Surgical conditions. Ear Nose Throat $J$ 1999; 78: 484.

2. GYOK, Yanghara N, SaikI T, HinOHRA Y. Chronic otitis media and tympanoplasty in aged patients. Auris Nasus Larynx 1990; 17: 14-8.

3. VARTIAINEN E, KARJALAINEN S. Surgery in elderly patients with chronic otitis media. Arch Aolaryngol Head Neck Surg 1985; 11: 509-10.

4. EMMETT J. Age as a factor in the success of tympanoplasty: A comparison of outcomes in the young and old. Ear Nose Throat J 1999; 78: 480.

5. RADPOUR S. Tympanoplasty in geriatric patients: Surgical conditions. Ear Nose Throat $J$ 1999; 78: 484-8.

6. NOMURA K, LINO Y, HASHIMOTO H, SUZUKI JI, KODERA K. Hearing results after tympanoplasty in elderly patients with middle ear cholesteatoma. Acta Aolaryngol 2001; 121: 919-24.

7. SAITO T, TANAKA T, TOKURIKI M, SHIBAMORI $\mathrm{Y}$, Yamamoto T, noda I, Omtsubo T, Saito $\mathrm{H}$. Recent outcome of tympanoplasty in the elderly. Otol Neurotol 2001; 22: 153-7.

8. PlazA E OTIEJ. Análisis crítico de las timpanoplastías. Rev Otorrinolaring 1980; 40: 11-6.

9. Vergara V, Aranaibia M, MaAsS J, OMsille M. Timpanoplastías: revisión de 4 años. Rev Otorrinolaringol Ar Cabeza Quello 2001; 61: 102-6.

10. VALLEJOS MP, DeNTONE L. Timpanoplastías en mayores de 50 años. Rev Otorrinolaringol Cir Cabeza Quello 2003; 63: 100-5.

11. Vergara V, Aranabia M, Corssen C, OMmsillem. Nuestro enfrentamiento a la patología de la otitis mediacrónica potencialmentepeligrosa Rev Otorrinolaringol Or Cabeza Ouello 2003; 63: 95-9. 